\title{
Dermoscopic findings of perifollicular pigmentation associated with vandetanib
}

\author{
Jade Cury-Martins ${ }^{1}$, Jose Antonio Sanches ${ }^{1}$
}

1 Department of Dermatology, University of São Paulo, São Paulo, Brazil

Key words: cutaneous adverse events, tyrosine kinase inhibitor, pigmentation, dermoscopy

Citation: Cury-Martins J, Sanches JA. Dermoscopic findings of perifollicular pigmentation associated with vandetanib. Dermatol Pract Concept. 2018;8(4):340-341. DOI: https://doi.org/10.5826/dpc.0804a20

Received: March 28, 2018; Accepted: May 7, 2018; Published: October 31, 2018

Copyright: @2018 Cury-Martins et al. This is an open-access article distributed under the terms of the Creative Commons Attribution License, which permits unrestricted use, distribution, and reproduction in any medium, provided the original author and source are credited.

Funding: None.

Competing interests: The authors have no conflicts of interest to disclose.

All authors have contributed significantly to this publication.

Corresponding author: Jade Cury-Martins, MD, PhD. Email: jadecury@yahoo.com.br

\section{Introduction}

Vandetanib is an oral tyrosine kinase inhibitor used in the treatment of different types of cancer. It acts by inhibiting the vascular and epidermal growth factor receptor and is associated with frequent adverse cutaneous reactions such as acneiform eruption, photosensitivity, pigmentary changes, exanthema, pruritus, and xerosis. We describe an unusual, peculiar form of perifollicular pigmentation associated with the use of the drug and show its dermoscopic findings.

\section{Case Presentation}

A 26-year-old male patient was diagnosed with metastatic medullary thyroid carcinoma. After combined treatment with surgery, radiotherapy, and chemotherapy, the disease progressed and vandetanib was started. Two weeks later, the patient developed an acneiform rash with lesions on the trunk and face and was subsequently treated with doxycycline $200 \mathrm{mg} / \mathrm{day}$ for 6 months with improvement. On dermatological follow-up, the patient was still taking vandetanib, with clearance of the acneiform lesions, but many pigmented follicular macules with a comedo-like appearance were noted on the face and chest (Figure 1). The pigmenta-

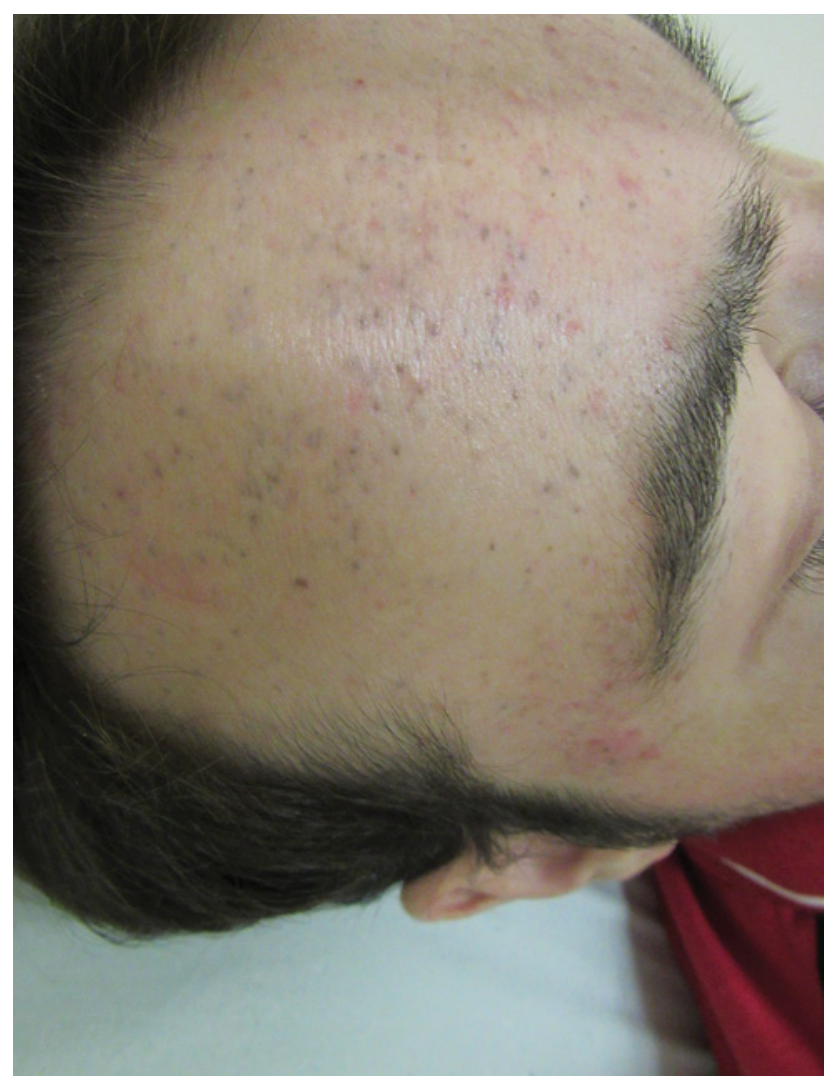

Figure 1. Pigmented follicular macules with comedo-like appearance on the face. [Copyright: (02018 Cury-Martins et al.] 
tion was apparently follicular; however, on dermoscopy, the blue-gray pigment was evident only in the perifollicular area with preservation of the center of the hair follicle (Figure 2). After orienting the patient on photoprotective measures, only a little improvement was noted. One year later, the patient had complete clearing, despite the continuous use of vandetanib.

\section{Discussion}

Among the pigmentary changes associated with vandetanib therapy, diffuse pigmentation in the exposed area is the most reported form. The blue-gray perifollicular pigmentation was rarely reported, and there are no similar reports of it with other agents of the same class. This seems to be specifically caused by this medication.

The pathogenesis of this peculiar pattern of pigmentation is not well established. Previous reports have demonstrated the histopathological alterations in a few similar cases revealing dermal iron and melanin deposition. The presence of pigmented macrophages and inflammatory infiltrate in the dermis was also documented [1]. Those findings correlate with the dermoscopy, with the blue-gray pigmentation corresponding to the presence of dermal pigment.

The previous use of doxycycline did not seem to factor into the etiology of this condition. Giacchero et al [2] showed that less than half of the patients in their series were treated with this medication. Prior photosensitivity preceding the pigmentation was described in two cases [1].

Definitive treatment of the lesions involves stopping the use of vandetanib followed by improvement after 3-6 months. However, recurrence occurs if the medication is reintroduced [2]. The use of topical depigmentation agents such as the combination of hydroquinone, tretinoin, and corticosteroids did not show benefits [1]. In a few cases,

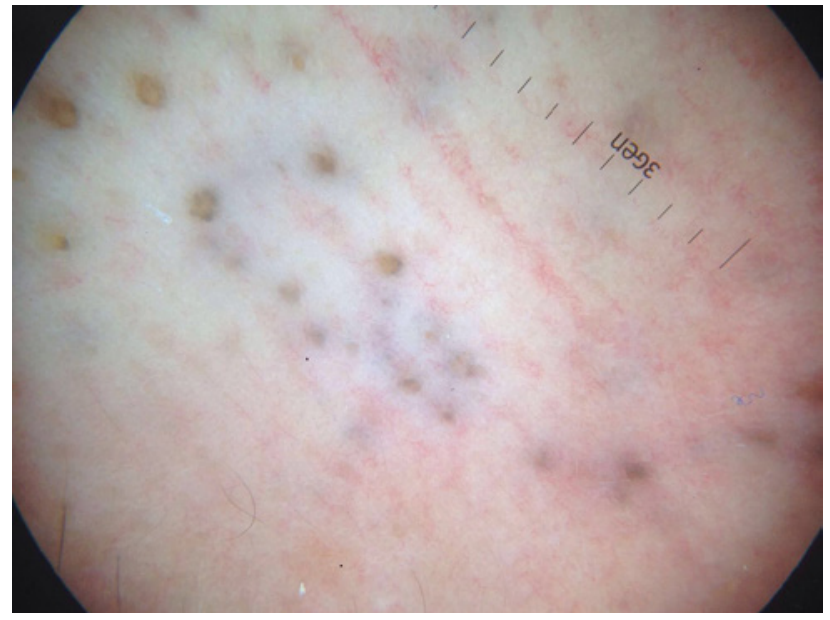

Figure 2. On dermoscopy, the blue-gray pigment is evident only on the perifollicular area. [Copyright: @2018 Cury-Martins et al.]

the use of photoprotective measures prevented worsening of the condition, [1].

\section{Conclusion}

In conclusion, we report the first description of dermoscopic findings of this rare pattern of perifollicular pigmentation associated with vandetanib use. As oncodermatology is involved in the supportive care of cancer patients, it is important for dermatologists to identify these possible adverse events and use dermoscopy as an additional tool.

\section{References}

1. Kong HH, Fine HA, Stern JB, Turner MLC. Cutaneous pigmentation following photosensitivity induced by vandetanib. Arch Dermatol. 2009;145(8):923-925.

2. Giacchero D, Ramacciotti C, Arnault JP, et al. New spectrum of skin toxic effects associated with the multikinase inhibitor vandetanib. Arch Dermatol. 2012;148(12):1418-1420. 